\title{
Chilenische Unternehmer im Außenhandel und Transportwesen der Provinz Coquimbo im 19. Jahrhundert
}

\author{
von Alf Zachäus
}

\begin{abstract}
From 1840 to 1870 , Chile's Norte Chico was the leading supplier of copper and silver for Europe and North America. Copper mines and smelters became the engine of a regional take off in the Chilean northern provinces Coquimbo and Atacama. Many historians, however, still consider that, in last instance, the prospering industry was controlled by foreign capital. In their view, above all British merchant banks controlled the trade, transports, and finances in the Chilean mining regions. But primary sources tell us another story. As the article shows, there was a core of modern Chilean capitalists who dominated the export as well as the railroad companies in the leading copper province Coquimbo.
\end{abstract}

\section{Der Topos Der einseitigen AbHängigkeit Auf DEM PrüFStAND}

Seit den Sechzigerjahren des letzten Jahrhunderts kam der Nationalgeschichte der Republik Chile im 19. Jahrhundert ein zentraler Stellenwert im transnationalen intellektuellen Diskurs über die Ursachen „,der Entstehung der Dritten Welt“ zu. Außerhalb Lateinamerikas spielte das klassische Werk der Dependencia-Schule von André Gunder Frank Capitalism and Underdevelopment aus dem Jahre 1967 für die Popularisierung des Fallbeispiels Chile innerhalb dieses Diskurses eine Schlüsselrolle. In den gleichen Genuss kam Brasilien, dessen Geschichte Gegenstand des zweiten Kapitels des Buches ist. Franks Wissen um und seine Urteile über die Historie des südamerikanischen Landes am Pazifik fußten zu einem guten Teil auf den Arbeiten des 
chilenischen Historikers Anibal Pinto Santa Cruz. ${ }^{1}$ Es muss hier nicht weiter ausgeführt werden, dass grundlegende Annahmen der Dependencia- und der Weltsystemtheorie schon des Öfteren mit Ergebnissen der jüngeren Forschung konfrontiert und relativiert oder gar völlig zurückgewiesen worden sind. ${ }^{2}$ Wesentliche Motive des von Pinto und Frank bereits in den 1960er Jahren gezeichneten Bildes der Geschichte Chiles im 19. Jahrhundert werden jedoch häufig bis heute unhinterfragt übernommen und harren immer noch ihrer Quellen gestützten Überprüfung.

Die Geschichte der postkolonialen chilenischen Ökonomie im 19. Jahrhundert verlief aus der Sicht der Historiographie in der Tradition Pintos und Franks in wenigen Sätzen zusammengefasst im Grunde wie folgt: Die sofortige Öffnung für den Außenhandel und für den Zustrom europäischen Kapitals nach den Unabhängigkeitskriegen und der Konsolidierung der jungen Republik bis 1830 ermöglichte Chile einen beispiellosen wirtschaftlichen Aufstieg. Dieses ,südamerikanische Wirtschaftswunder" manifestierte sich vor allem in dem take off der Kupfer- und Silbermontanindustrie in den Provinzen Coquimbo und Atacama, dem Norte Chico. Die Kupferdistrikte Tamaya und La Higuera in Coquimbo stiegen zu den größten Produzenten des roten Metalls im mittleren Drittel des 19. Jahrhunderts auf, die Silberminen in Atacama spielten nach der Entdeckung der Lagerstätten am Cerro Blanco 1832 auf ihrem Zielmarkt eine vergleichbare Rolle. ${ }^{3}$ Sie behielten ihre Stellung als industrielle Leitsektoren bis zum Pazifikkrieg 1879-1883, in dessen Folge die Salpeterindustrie im gewaltsam von der Republik inkorporierten Großen Norden ihr Erbe antrat. Trotz aller beeindruckenden Industrialisierungsbemühungen lag das Schicksal der Montanindustrie im Norte Chico allerdings auch in den Dekaden ihrer größten Erfolge von vornherein in den Händen nordatlantischer, vor allem britischer Merchant Banken, so die tradierte

1 André Gunder Frank, Capitalism and Underdevelopment in Latin America. Historical Studies of Chile and Brasil (New York/London 1967), S. 48-56; und Anibal Pinto Santa Cruz, Chile, un caso de desarollo frustrado (Santiago de Chile 1962).

2 Siehe u. a. Hartmut Elsenhans, Abhängiger Kapitalismus oder bürokratische Entwicklungsgesellschaft: Versuch über den Staat in der Dritten Welt (Frankfurt am Main 1984); Cornelius Torp, „Die Weltsystemtheorie Immanuel Wallersteins: eine kritische Analyse“: Jahrbuch für Wirtschaftsgeschichte 1998, 1 (1998), S. 217 f.

Leland R. Pederson, The Mining Industry of the Norte Chico, Chile (Evanston, IL 1966), S. 180. 
Auffassung. Autoren wie John Mayo oder Eduardo Cavieres zufolge funktionierten Produktion und Distribution im Norte Chico in wenigen Worten zusammengefasst nach folgendem grundlegenden Schema: Chilenen bewerkstelligten die Produktion, Briten vermittelten die Aufträge, stellten die nötigen Kredite bereit und organisierten den Transport per Eisenbahn und Schiff. Die Abhängigkeit der einheimischen mineros von kurzfristigen Krediten perpetuierte dabei deren Status als ewige Schuldner, die von der Hand in den Mund lebten. ${ }^{4}$

Erwähnt werden muss hier, dass in einer Reihe jüngerer Arbeiten die These von der technologischen Rückständigkeit der Montanindustrien im Chile der Jahrzehnte des Laissez-faire-Kapitalismus zunehmend in Frage gestellt wird. So stellen Autoren wie Luis Ortega oder Ricardo Nazer Ahumada die technische Ebenbürtigkeit der chilenischen Kohlen- bzw. Kupfermontanindustrie verglichen mit den fortgeschrittensten Industrien in Großbritannien heraus. Das Gleiche gilt für die ingenieurtechnischen wie kaufmännischen Fähigkeiten von schillernden Unternehmerpersönlichkeiten wie José Tomás Urmeneta oder Joaquín und Agustín Edwards Ossandón. Aber selbst in diesen jüngeren Arbeiten in der Tradition der Wirtschaftsregionalgeschichte Sidney Pollards bzw. der Business History Alfred Chandlers kommt der Topos einseitiger äußerer Abhängigkeit nicht wirklich auf den Prüfstand. ${ }^{5}$

Im Folgenden soll die Gültigkeit dieser These für den Export von Montanprodukten und den Eisenbahnsektor der Provinz Coquimbo näher untersucht werden. Die unmittelbare, wenn man so will schicksalhafte, Verflechtung des chilenischen Außenhandels mit Montanprodukten mit den Märkten in Europa und Nordamerika manifestierte sich in den dieses Geschäft von Chile aus betreibenden Netzwerken.

Am spanisch-amerikanischen Kolonialhandel konnten Nichtspanier nur über spanische Mittelsmänner partizipieren. Über diese Form des intermediären Handels bildeten sich ab dem 17. Jahrhundert Netzwerke heraus, in denen die Spanier das Kommissionsgeschäft für die Ausländer abwickelten. Der direkte Warenverkehr lag in der Regel in den Händen spanisch-amerikanischer Familien. Obwohl auf diese

4 John Mayo, British Merchants and Chilean Development, 1851-1886 (London 1987), S. 23; und Eduardo Cavieres, Comercio chileno y comerciantes ingleses, 1820 1880. Un ciclo de historia económica (Santiago de Chile 1999).

5 Luís Ortega, "The First Four Decades of Chilean Coal Mining Industry, 18401879": Journal of Latin American Studies 14, 1 (1982), S. 1-32. Ricardo Nazer Ahumada, José Tomás Urmeneta. Un empresario de minería del siglo XIX (Santiago de Chile 1993). 
Weise der Aufbau eigener Netzwerke von Nichtspaniern im kolonialen Spanisch-Amerika unterblieb, gelang es ihnen in Chile nach dem Wegfall des spanischen Handelsmonopols innerhalb kürzester Frist im Außenhandel Fuß zu fassen und vor Ort ein dichtes Beziehungsgeflecht zu etablieren. ${ }^{6}$

Wie in den anderen Hafenstädten der jungen südamerikanischen Republik, über die die Aus- und Einfuhr von Gütern gestattet war, bildete sich auch in Coquimbo schon in den ersten drei Jahrzehnten des 19. Jahrhunderts eine transnationale Gesellschaft von Händlern heraus. Diese betrieben entweder auf eigene Rechnung oder als Agenten von Merchant Banken, die in London, Liverpool oder Glasgow, Boston, Baltimore oder Hamburg ihren Hauptsitz hatten, ihre Geschäfte. ${ }^{7}$

Bis zum Ende des 19. Jahrhunderts behielt das Kommissionsgeschäft beim Verkauf von Produkten der chilenischen Montanindustrie eine große Bedeutung. Typisch war der folgende Vertrag zwischen Antony Gibbs \& Sons und Urmeneta \& Errázuriz vom 11. April 1879:

„Die Modifikationen sind die folgenden: Die garantierte Kommission soll 2 anstatt $3 \%$ brutto betragen, anstatt 300.000 werden pro Jahr $333.333 £$ als Minimum oder Kommission verschickt: mit inbegriffen sind garantierte $0,5 \%$ Maklergebühr, anstatt 9.000 sollen es $6.666 £$ Nettosumme mit einer Garantie von $5.000 £$ sein. Sollte unsere Nettokommission in keinem Jahr bis zum 31. Dezember der von Urmeneta Errázuriz gewünschten Nettosumme auf seinem Debitkonto entsprechen, so behalten wir uns vor, unser Äquivalent mit einem Zinsfuß von $10 \%$ bezogen auf eine Kreditsumme von $100.000 £$ pro Jahr zu versichern. Die Kommission für die Rücksendung soll anstatt $25 \%$ bezogen auf eine Summe von $9.000 £ 50 \%$ oder in Nettosumme $5.000 £$ betragen als vierter Satz im beigefügten Vertrag. " 8

Trotz der großen Bedeutung, die Kommissionsgeschäfte dieser Art bis zum Niedergang der Montanindustrie im Norte Chico in den 1890er Jahren behielten, sollte die Rolle des kurzfristigen Kredites im Kupferund Silbersektor auch nicht überschätzt werden. In der Montanindustrie

6 Siehe u. a. Renate Pieper, „Kolonialhandel und Weltmarktintegration“: Friedrich Edelmayer/Bernd Hausberger/Michael Weinzierl (Hg.): Die beiden Amerikas. Die Neue Welt unter kolonialer Herrschaft (Frankfurt am Main 1996), S. 141-158, hier: S. 149. $\mathrm{Zu}$ der Größe des und der Rangfolge der Häfen im Handel mit den spanischen Kolonien siehe u. a. John Fischer, "El comercio español con América, 1797-1820”: Sarabia Viejo (Hg.), IX. Congreso de Historia de América: Europa e Iberoamérica: Cinco siglos de intercambios, 3 Bände (Sevilla 1992), Bd. 2, S. 693-703.

7 Mayo, British Merchants (Anm. 4), S. 15-17.

8 Guildhall Library, Antony Gibbs \& Sons, Private Letters, Ms 11, 470/3, Brief von William Gibbs, Valparaíso, April 1879. 
in Coquimbo lässt sich das Gewicht von Fremdkapital im Bergbau für die Jahre 1871, 1872 und 1874 anhand der Statistischen Jahrbücher der Republik Chile, die für die 1870er und 80er Jahre detaillierte Montanstatistiken beinhalten, etwas genauer abschätzen. Nach den Angaben der amtlichen Statistik der Republik wurden 1871 in Coquimbo 172 Kupfer- und 21 Silbergruben mit eigenem Kapital betrieben. 79 Kupfer- und 10 Silbergruben waren von fremdem Kapital abhängig. 1872 kamen auf 177 mit eigenem Kapital arbeitende Kupfer- und Silbergruben 21 mit Hilfe fremden Kapitals betriebene Kupferminen. 1874 kamen auf 208 mit eigenem Kapital arbeitende Kupfergruben 73 von fremdem Kapital abhängige. ${ }^{9}$

Rund zwei Drittel der Besitzer von Bergwerken in der Provinz Coquimbo betrieben den Angaben der Statistischen Jahrbücher für 1871, 1872 und 1874 zufolge ihre Betriebe also ohne Kredite in Anspruch genommen zu haben. Obwohl das kurzfristige Darlehen eine bedeutende Rolle bei der Finanzierung des laufenden Betriebes im Bergbau hatte, kann kaum davon die Rede sein, dass die Mehrheit der Gruben über diese Form der Vorfinanzierung am Laufen gehalten wurde.

Noch deutlicher tritt der Widerspruch zwischen unhinterfragten Grundannahmen und den überlieferten Quellen im Außenhandel hervor. Obwohl auch in Chile Korruption bei Zollbehörden und Schmuggel keine unbekannten Phänomene waren, kann den in den Akten der Provinzialverwaltungen und des Finanzministeriums überlieferten Daten ein hohes Maß an Realitätsnähe zugesprochen werden. ${ }^{10}$ Für diese Annahme spricht, dass die lokalen Behörden zum großen Teil aus diesen Einnahmen finanziert wurden, also an einem gut funktio-

9 Vgl. die Ausgaben des Anuario estádistico de la República de Chile (Santiago de Chile) für die Jahre 1871, 1872 und 1874.

${ }^{10}$ Nach dem Zollgesetz von 1858 waren Ancud, Valdivia, Talcahuano, Tomé, Constitución, Valparaíso, Coquimbo, Huasco und Caldera (Art. 1) Exporthäfen. In diesen Häfen existierten Zollstationen. Mit Inkrafttreten des Zollgesetzes vom 31. Oktober 1864 (Art. 1) nahmen in Milipulli, Coronel und Mejillones ebenfalls Zollstationen ihren Betrieb auf, die von Exporteuren angelaufen werden konnten. Ab 1872 durften dann auch über die Häfen Carrizal Bajo und Chañaral de las Animas Waren ein- und ausgeführt werden. Der Zollbehörde von Coquimbo untergeordnete Häfen waren laut dem Zollgesetz von 1872 (Art. 4) Tongoy, Guayacán und Tortoralillo und die Zollstationen in den Anden an der argentinischen Grenze Elquí und Ovalle, Huanto, Calderón und Cuncumen. Die Pazifikhäfen von Pichitangui und Vilos im Süden der heutigen IV. Region, waren hingegen der Zollbehörde von Valparaíso untergeordnet. 
nierenden Zollsystem interessiert sein mussten. ${ }^{11}$ So konnten Luz María Mendéz Beltrán und ihre Mitarbeiter anhand der in den Aktenbeständen verschiedener Ministerien und Provinzialverwaltungen überlieferten Statistiken und Einzelrechnungen unter anderem die Höhe des Exportes von Kupfer über die Zollstation von Coquimbo für die Jahre von 1800 bis 1840 ermitteln. Ab 1865 sind die Statistiken der Zollverwaltungen bezüglich der Ausfuhr von Kupfer mit größerer Vorsicht zu genießen, da am 7. Oktober 1865 die Zölle aufgehoben wurden und die Statistiken ab dann erhebliche Lücken aufweisen. ${ }^{12}$

Die Untersuchungen von Méndez Beltrán belegen, dass sich bereits seit dem Ende der Befreiungskriege ein Netzwerk von Händlern und Bankiers im Handel mit Mineralien aus dem Kleinen Norden etabliert hatte, in dem Angelsachsen dominierten. In Coquimbo saßen in den 1820 er bis 1840er Jahren 206 Exporteure von Mineralien. 13 von ihnen waren relevant und exportierten $75 \%$ aller aus der Provinz ausgeführten Erze. Es handelte sich um Samuel Haviland (37\%), Alfred Cobb (5\%), George Edwards (5\%), Daniel W. Frost (4\%), Thomas Smith (4\%), die Brüder Walker (4\%), Edwards und Stewart (3\%), Thomas Kendall (2\%), Joaquín Vicuña (2\%), Alexander Caldleugh (2\%), George Edwards (2\%), José A. Subercaseaux (2\%) und David Ross Gillespie ( $2 \%){ }^{13}$

11 Luz María Méndez Beltrán, La exportación minera (Santiago de Chile 2004), S. 62.

12 Archivo Nacional, Ministerio de Hacienda, Vol. 636, 1868.

13 Die Brüder Walker hatten Niederlassungen in Coquimbo, Huasco und Valparaíso. John Walker kam 1820 nach Chile und war der Sohn des Oberbürgermeisters von Birmingham. Samuel Haviland, der ebenfalls in Coquimbo residierte, war US-Amerikaner und Diego Carvallo, selbst Einwohner von Copiapó, kam ursprünglich aus der Gegend von Valparaíso. Thomas Smith war Engländer, und der seit 1820 im Kleinen Norden Geschäfte betreibende David Ross Guillespie Schotte; Thomas Kendall war aus Neuseeland nach Chile eingewandert. Der in London geborene Alexander Caldcleugh hatte eine Ausbildung als Mineraloge genossen und besaß bis 1836 Gruben in Ovalle. John Sewell und Thomas Eastman White waren gebürtige Briten. Whites Cousin Adolf Eastman Quiroga studierte in Sachsen Mineralogie, heiratete später Manuela Urmeneta und wurde der Verwalter der Gruben José Tomás Urmenetas in Tamaya. Vgl. Méndez Beltrán, Exportación minera (Anm. 11), S. 158-174. 
TABelle 1: Exporteure von Silber

\begin{tabular}{l|r|r}
\hline Exporteur & Tonnen & Prozent \\
\hline Brüder Walker & 24,79 & 7,23 \\
Haviland, Samuel F. & 23,30 & 6,81 \\
Cienfuentes, José & 12,79 & 3,74 \\
Verschiedene Bewohner Coquimbos & 12,69 & 3,71 \\
Carvallo, Diego & 12,44 & 3,64 \\
Gallo, José María & 10,64 & 3,11 \\
Bringas und Sánchez & 8,40 & 2,46 \\
Edwards, George & 8,03 & 2,35 \\
Videla, Francisco & 7,25 & 2,12 \\
Infante, Buenaventura & 6,41 & 1,87 \\
Edwards Ossandón, Agustín & 5,58 & 1,63 \\
Haviland und Smith & 4,66 & 1,36 \\
Ossa, Francisco & 4,34 & 1,27 \\
\hline Summe & 141,32 & 41,32 \\
& $(=614.452$ & \\
\hline
\end{tabular}

Quelle: Luz María Méndez Beltrán, La exportación minera (Santiago de Chile 2004), S. 158.

Wichtige Exporteure von Kupfer aus dem Kleinen Norden in den ersten vier Jahrzehnten des 19. Jahrhunderts waren Samuel F. Haviland (10.513 t, $14 \%$ des Gesamtexports von Kupfer), die Brüder Walker (3.493 t, 5\%), George Bingley (2.666 t, 3,5\%), George Edwards (2.635 t, 3,5\%), José Miguel Almarza (1.882 t, 3\%), Brian Adams (1.882 t, 2,5\%), Sewell und Patrickson (1.561 t, 2\%), Alfred Cobb (1.201 t, $2 \%)$, Edward Abbot (1.201 t, 2\%), McNail Price und Co. (1.161 t, 1,5\%), Daniel W. Frost (1.155 t, 1,5\%), Thomas Smith (1.067 t, 1\%), Carlos Ferrari (1.040 t, $1 \%$ ) und Agustín Edwards (923 t, $1 \%$ ). Diese Exporteure führten zwischen 1800 und 184033.236 t oder $44 \%$ allen chilenischen Kupfers aus. Fünf von ihnen waren Briten, zwei Nordamerikaner, vier Chilenen, einer Italiener, die Herkunft der zwei letzten konnte nicht geklärt werden. Einer der Chilenen, George Edwards, war gebürtiger Brite, der als Schiffsarzt nach Chile gekommen und per Heirat Oberhaupt einer anglo-chilenischen Familiendynastie geworden war. Zuvor war er zum Katholizismus konvertiert. Bei Agustín Edwards Ossandón handelte es sich um einen seiner Söhne, der zu einem der erfolgreichsten Merchant Bankiers in Chile 
aufstieg. ${ }^{14}$ Anders verhielten sich die Dinge bei der Ausfuhr von Silber. Bei den 13 größten Silberhändlern handelte es sich um acht Chilenen, zwei US-Amerikaner, zwei Briten und um eine Gruppe von Händlern mit Wohnsitz in Coquimbo, deren Herkunft unbekannt ist. ${ }^{15}$

Wie oben bereits erwähnt, erlebte der Kleine Norden im mittleren Drittel des 19. Jahrhunderts einen regional begrenzten Industrialisierungsschub, dessen Schrittmacher neu errichtete Hüttenwerke waren, die mit der sogenannten englischen Methode produzierten. Den Anfang machte der elsässische Berg- und Hütteningenieur Charles Lambert, der das erste Werk dieses Typs $1830-1832$ in La Serena errichten ließ. In den folgenden drei Dekaden verwandelte sich ein verschlafener kleiner Pazifikhafen nach dem anderen zu einem Standort modernster Kupferschmelzen. Die Mehrheit dieser Werke befand sich im Besitz einer neuen Schicht chilenischer Industriebourgeois, die ihren britischen Vorbildern nicht nur in jeder Hinsicht nacheiferten, sondern ob ihrer Erfolge und fachlichen Kompetenzen auch in Europa großes Ansehen genoss. Eine nähere Untersuchung der Statistiken der Zollbehörde von Coquimbo belegt, dass es eben jenen Geschäftsleuten gelang, die Ausfuhr der Provinz Coquimbo bereits in den Jahren von 1841 bis 1852 zunehmend zu dominieren. In den monatlichen bzw. vierteljährlichen Bilanzen des Zollamtes von Coquimbo erscheint in den Jahren nach 1840 eine Gruppe von insgesamt 88 Exporteuren, Kaufleuten und Montanunternehmern, die wenigstens in einem Jahr Zölle von mehr als 100 Pesos zu entrichten hatten, wobei die Fluktuation sehr groß war: Viele der Namen tauchen nur in wenigen, manche nur in einem oder nur zwei Jahren in der Statistik auf. 1841 lag der von diesen Geschäftsleuten an die Zollbehörde von Coquimbo zu entrichtende Zoll bei insgesamt 40.976, 1851 bei $61.759,1852$ allerdings nur noch bei 1.987 Pesos. ${ }^{16}$

${ }^{14}$ In den ersten beiden Jahrhunderten der Kolonialzeit wurden die Gruben in den Vizekönigreichen Neuspanien und Peru von Aristokraten betrieben, die häufig hohe militärische und öffentliche Ämter in den Kolonialbehörden bekleideten. Im 18. Jh. änderte sich das Bild. Der Bergbau wurde zunehmend zum Feld der neuen kolonialen mestizischen Mittelschichten. Der typische Betreiber einer Grube in einer spanischen Kolonie war nun der Besitzer eines Landgutes von geringer Ausdehnung. Vgl. Sergio Villalobos, Origen y ascenso de la burguesia chilena (Santiago de Chile 1987), S. 20 und 48.

15 Méndez Beltrán, La exportación minera (Anm. 11), S. 157.

16 Von 1851 bis 1853 sah das Zollgesetz von 1851 eine sukzessive Verringerung des Satzes von $4 \%$ auf Barrenkupfer und Kupfererz bis auf Null vor. Mit dem Zollgesetz von 1858 führte man dann jedoch wieder ein Zollsatz von $5 \%$ ein. 1864 setzte man 
Unter den 88 Händlern mit einer jährlichen Mindestverbindlichkeit von 100 Pesos gegenüber der Zollbehörde von Coquimbo lässt sich eine Gruppe von zwölf Händlern herauskristallisieren, die in den Jahren von 1841 bis $1852 \mathrm{im}$ arithmetischen Mittel 83\% der Verbindlichkeiten zu begleichen hatten. Zu ihnen gehörten Gabriel Menoyo, José R. Astaburuaga, Jorge Joaquín und Agustín Edwards Ossandón, Charles Lambert, Hugo Cogrove, José Monreal, Diego C. Bräuninger, Samuel Haviland, David Ross, Robert Alison und ein weiterer Händler namens Plummer.

Die bereits von Méndez Beltrán erwähnten Haviland und Ross sowie Plummer tauchen in der Statistik jedoch nur noch in den Jahren von 1841 bis 1846 auf, Bräuninger nur von 1847 bis 1853. Der Anteil von Charles Lambert und den Brüdern Joaquín und Agustín Edwards an den Verbindlichkeiten der Zollbehörde lag im arithmetischen Mittel bei $34 \%$, von 1848 bis 1850 sogar bei über $50 \%$. Charles Lambert kam 1845 auf einen Anteil von $27 \%$ und 1850 auf $33 \%$, im Durchschnitt hatte er $17 \%$ aller Verbindlichkeiten bei der Zollbehörde von Coquimbo zu begleichen. Joaquín und Agustín

diesen Satz auf $3 \%$ des Warenwertes herab. Im Zollgesetz von 1851 wurde ein Ausfuhrzoll von 4\% des Marktwertes auf Kupferbarren und -schienen, rohes und gebranntes Kupfererz für das Jahr 1851 festgesetzt. Dieser Satz sollte bis 1852 auf $2 \%$ herabgesetzt werden. Ab 1853 war der Export von Kupfer zollfrei (Art. 3). Laut Artikel 4 stand den Munizipien eine Exportgebühr von 1,5\% zu. Ab 1858 belegte man die Ausfuhr von Silber, Barrenkupfer und -silber, Kupfer- und Silberprofilen, reinem Feinsilber oder Silberlegierungen und Silbererzen jedoch wieder mit einem Zollsatz von $5 \%$ (Art. 58). Das Zollgesetz von 1864 (Art. 31) legte einen Satz von 3\% für die Ausfuhr von Barrenkupfer und Kupferprofilen fest (Art. 2). Auf die Ausfuhr von $100 \mathrm{~kg}$ Stangenkupfer wurden 65 Centavos erhoben (Art.4), auf $100 \mathrm{~kg}$ reines Silbererz oder Silberlegierungen 1 Peso (Art. 3). Der Zollsatz für Rohkupfer lag bei 30 Centavos pro $100 \mathrm{~kg}$ (Art. 5). Die Montanunternehmer der Provinz von Coquimbo konnten 1869 im Abgeordnetenhaus den Antrag durchbringen, dass sie keine weiteren Abgaben zusätzlich zu dem im Zollgesetz von 1864 festgelegten Zollsatz für den Export von Kupfer zu zahlen hatten. Im Senat wurde dieser Vorschlag am 6. Dezember 1869 angenommen. Vgl. Sesiones del Senado de la República de Chile 1869 (archiviert in der Biblioteca Nacional de Chile), S. 23. Mit Inkrafttreten des Zollgesetzes vom 26. Dezember 1872 (Artikel 40 Absatz 3) wurde ein Zollsatz für die Ausfuhr von Barrenkupfer und Kupferprofilen von 60 Centavos pro spanischen Zentner (46 kg) festgelegt. Die Ausfuhr von Kupfererzen mit einem Gehalt von bis zu $1 \%$ wurde für zollfrei erklärt. Höherwertige Erze belegte man mit einem Zollsatz von 90 Centavos für jedes Prozent über der Einprozentmarge pro spanischem Zentner Kupfererz (Abs. 4). Der Zollsatz für Exporte von Barrenkupfer und gewalztem Kupfer lag bei 60 Centavos pro Zentner. Die Ausfuhr von Rohkupfer mit bis zu $10 \%$ Metallgehalt wurde für zollfrei erklärt (Art. 2) Für jeden Prozentpunkt, der diese Marge überschritt, sollten 90 Centavos pro Zentner bezahlt werden (Art. 3). 
Edwards kamen 1847 auf $28 \%$ und 1848 auf 29\%, durchschnittlich lag ihr Anteil bei $20 \%$ (siehe Diagramm 1 und Tabelle 2).

Nach den von Charles Lambert sowie Joaquín und Agustín Edwards Ossandón zu entrichtenden Zöllen lag der Wert des von beiden Unternehmen exportierten Kupfers in den Jahren von 1841 bis 1852 bei durchschnittlich 130.290 Pesos und im Falle Lamberts bei 144.549 Pesos. 1842 erreichten die Exporte von Lambert einen Wert von 29.355, 1851 lag er bereits bei 247.150 Pesos. Die Brüder Edwards exportierten 1841 Kupfer im Wert von 44.033 Pesos, 1851 erreichte ihr Export den Wert von 113.433 Pesos. Der Gouverneur des Bezirkes von Ovalle, Francisco Bascuñán Guerrero, setzte in einem Bericht von 1848 den Wert der 1846 aus der Provinz Coquimbo ausgeführten Menge Kupfer in Form von Kupfererz, -barren und -halbzeugen mit 400.000 Pesos an. ${ }^{17}$ Legt man die Daten aus den Büchern der Zollstation von Coquimbo zugrunde, so lag der Anteil der Ausfuhren Lamberts und beider Brüder Edwards bereits 1846 mit 199.777 Pesos bei knapp der Hälfte des Gesamtexportes der Provinz (siehe Diagramm 2 und Tabelle 3).

Diagramm 1: Anteile von Charles Lambert, Joaquín und Agustín Edwards Ossandón, Ramón Ovalle und URmeneta ERRÁZURIZ AN DEN ZOLLEINNAHMEN DER ZOLLSTATION VON Coquimbo in Prozent, 1841-1863

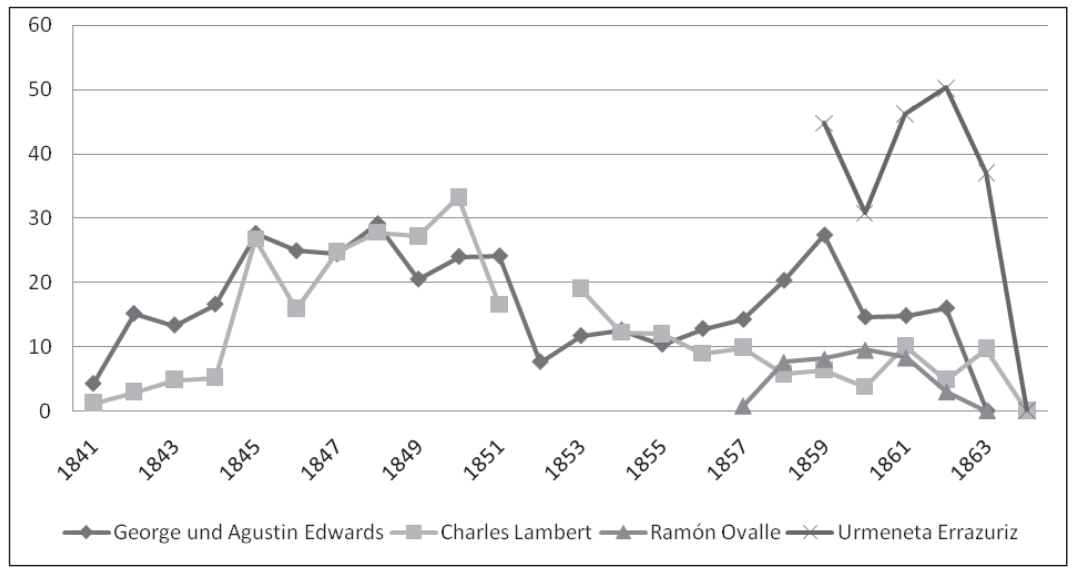

Quelle: Archivo Nacional, Ministerio de Hacienda.

${ }_{17}$ Vgl. Archivo Nacional, Intendencia de Coquimbo, Vol. 252, 1848-1849. 
Tabelle 2: Anteile von Joaquín und Agustín Edwards Ossandón, Charles Lambert, Ramón Ovalle und URMENETA ERRÁZURIZ AN DEN EINNAHMEN DER ZOLLSTATION VON COQUimbo

\begin{tabular}{|c|c|c|c|c|}
\hline Jahr & $\begin{array}{l}\text { Joaquín und } \\
\text { Agustín } \\
\text { Edwards (\%) }\end{array}$ & $\begin{array}{l}\text { Charles } \\
\text { Lambert }(\%)\end{array}$ & $\begin{array}{l}\text { Ramón } \\
\text { Ovalle (\%) }\end{array}$ & $\begin{array}{l}\text { Urmeneta } \\
\text { Errázuriz (\%) }\end{array}$ \\
\hline 1841 & & 1 & & \\
\hline 1842 & 4 & 3 & & \\
\hline 1843 & 15 & 5 & & \\
\hline 1844 & 13 & 5 & & \\
\hline 1845 & 17 & 28 & & \\
\hline 1846 & 28 & 16 & & \\
\hline 1847 & 25 & 25 & & \\
\hline 1848 & 25 & 28 & & \\
\hline 1849 & 29 & 27 & & \\
\hline 1850 & 20 & 33 & & \\
\hline 1851 & 24 & 17 & & \\
\hline 1852 & 24 & & & \\
\hline 1853 & 8 & 19 & & \\
\hline 1854 & 12 & 12 & & \\
\hline 1855 & 13 & 12 & & \\
\hline 1856 & 10 & 9 & & \\
\hline 1857 & 13 & 10 & 1 & \\
\hline 1858 & 14 & 6 & 8 & \\
\hline 1859 & 20 & 6 & 8 & 45 \\
\hline 1860 & 27 & 4 & 10 & 31 \\
\hline 1861 & 15 & 10 & 8 & 46 \\
\hline 1862 & 15 & 5 & 3 & 50 \\
\hline 1863 & 16 & 10 & & 37 \\
\hline
\end{tabular}

Quelle: Archivo Nacional, Ministerio de Hacienda, Vol. 186, 192, 193, 206, 207, 224, $225,228,245,246,287,328,356,382,398,422,443,473,508$ und 536 
Tabelle 3: Wert des verzollten Kupfers und Silbers von Joaquín und Agustín Edwards Ossandón, Charles Lambert, Ramón Ovalle und Urmeneta Errázuriz zu laufenden Preisen, in chilenischen Pesos

\begin{tabular}{c|c|c|c|c}
\hline Jahr & Brüder Edwards & $\begin{array}{c}\text { Charles } \\
\text { Lambert }\end{array}$ & $\begin{array}{c}\text { Ramón } \\
\text { Ovalle }\end{array}$ & $\begin{array}{c}\text { Urmeneta } \\
\text { Errázuriz }\end{array}$ \\
\hline 1841 & & 5.600 & & \\
1842 & 29.355 & 20.378 & & \\
1843 & 84.289 & 27.033 & & \\
1844 & 78.410 & 30.433 & & \\
1845 & 94.333 & 101.289 & & \\
1846 & 190.255 & 109.522 & & \\
1847 & 180.333 & 178.978 & & \\
1848 & 195.022 & 221.655 & & \\
1849 & 271.800 & 253.367 & & \\
1850 & 197.800 & 320.867 & & \\
1851 & 247.150 & 113.433 & & \\
1852 & 31.933 & & & \\
1853 & 266.067 & 661.733 & & \\
1854 & 523.400 & 547.400 & & \\
1855 & 770.800 & 734.200 & & \\
1856 & 571.800 & 493.667 & & \\
1857 & 887.733 & 684.933 & 53.808 .500 & \\
1858 & 1.011 .200 & 410.067 & 539.933 & \\
1859 & 241.883 & 151.333 & 193.400 & \\
1860 & 410.467 & 112.467 & 285.933 & \\
1861 & 339.950 & 470.700 & 387.567 & \\
1862 & 367.350 & 242.733 & 148.066 & \\
1863 & 368.700 & 446.733 & & \\
\hline & & & \\
\hline
\end{tabular}

Quelle: Archivo Nacional, Ministerio de Hacienda, Vol. 186, 192, 193, 206, 207, 224, $225,228,245,246,287,328,356,382,398,422,443,473,508$ und 536

Von 1853 bis 1864 setzte sich der Konzentrationsprozess im Außenhandel analog zu jenem in der Montanindustrie in Coquimbo fort. Die große Gruppe von Geschäftsleuten, die in den ersten vier Jahrzehnten des 19. Jahrhunderts die Ausfuhr der Provinz dominiert hatte, spielte nun eine immer geringere Rolle. Während die bereits erwähnten Haveland, Ross und Bräuninger nicht mehr in der Zollstatistik auftauchen, mussten in diesen Zeitraum die Hüttenunternehmer Urmeneta 


\section{Diagramm 2: Wert des verzollten Kupfers und Silbers von Joaquín und Agustín Edwards Ossandón, Charles Lambert, Ramón Ovalle und Urmeneta ERrázuriz zu laufenden Preisen, 1841-1863}

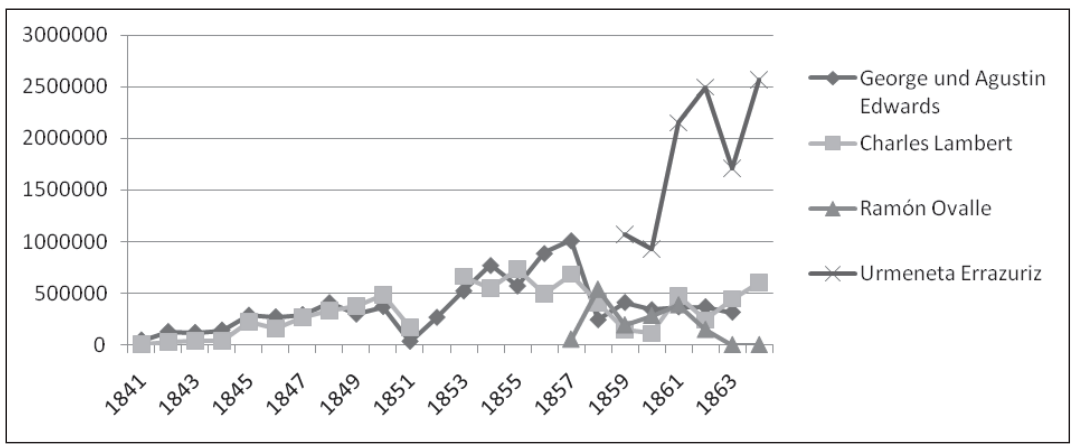

Quelle: Archivo Nacional, Ministerio de Hacienda, Vol. 186, 192, 193, 206, 207, 224, 225, 228, 245, 246, 287, 328, 356, 382, 398, 422, 443, 473, 508 und 536.

Errázuriz (1859-1864) und Ramón Ovalle (1857-1862) über mehrere Jahre hinweg größere Beträge an Ausfuhrzöllen entrichten. Der Gruppe der zwölf größten Exporteure waren nach wie vor durchschnittlich $81 \%$ aller Verbindlichkeiten zuzuordnen. Die Brüder Edwards Ossandón, Charles Lambert, Urmeneta Errázuriz und Ramón Ovalle bezahlten im arithmetischen Mittel $51 \%$ aller Ausfuhrzölle bei der Zollbehörde von Coquimbo. Auf Lambert entfielen im Durchschnitt 10\%, auf die Edwards $15 \%$, auf Ovalle in den Jahren 1857 bis $18626 \%$ und auf Urmeneta Errázuriz von 1859 bis 1864 64,5\% aller Ausfuhrzölle. José Tomás Urmeneta und Maximiano Errázuriz waren somit bereits 1859, im ersten Jahr, in dem beide noch getrennt in den Büchern der Zollbehörde von Coquimbo geführt wurden, in die kleine Gruppe der den Außenhandel der Provinz Coquimbo dominierenden Unternehmer aufgestiegen. ${ }^{18}$

${ }^{18}$ Lambert exportierte in den Jahren 1853 bis 1864 Kupfer und Silber im Wert von durchschnittlich 463.203 Pesos zu laufenden Preisen. 1852 waren es 661.733, 1864 waren es 602.466 Pesos. Edwards führte Kupfer mit einem durchschnittlichen Wert von 506.296 Pesos aus. Urmeneta \& Errázuriz exportierten in den Jahren 1859 bis 1864 Barrenkupfer mit einem durchschnittlichen Wert von 1.819.467 Pesos. Ovalle führte 1857 Kupfer im Wert von 53.500 Pesos aus, 1862 besaß sein Export einen Wert von 148.067 Pesos zu laufenden Preisen. Durchschnittlich lag der Wert der von ihm exportierten Menge Kupfer bei 268.116 Pesos. Archivo Nacional, Ministerio de Hacienda, Vol. 246, 287, 328, 356, 382, 398, 422, 443, 473, 508 und 536, 1852-1864. 
Angesichts der hier ausgewerteten Quellen lässt sich die These von einer einseitigen Abhängigkeit vom europäischen, vor allem britischen Handels- und Finanzkapital für die Außenwirtschaft der Kupfermontanregion Coquimbo nicht mehr aufrechterhalten. Coquimbos Export wurde spätestens ab 1845 größtenteils von wenigen fortgeschrittenen Montanunternehmen realisiert, die, abgesehen von Charles Lamberts Hüttenwerk in La Serena, in den Händen chilenischer Staatsbürger waren.

Die bis heute weit verbreitete Vorstellung, der Absatz und die Finanzierung der Montanindustrie im Kleinen Norden hätten im 19. Jahrhundert ausschließlich in nichtchilenischen Händen gelegen, muss also relativiert werden. Beim Güterexport bedarf sie der Revision.

\section{BESITZVERHÄLTNISSE BEI DEN EISENBAHNGESELLSCHAFTEN}

Seit 1862 wurden die Hüttenwerke in La Serena, Coquimbo und Guayacán über die Linie der Eisenbahngesellschaft von Coquimbo (Compañia Ferro-Carril de Coquimbo) beliefert, die sie mit den Abbaugebieten im Hinterland verband. ${ }^{19} 1867$ nahm die Compañia Ferro-Carril de Tongoy den Betrieb auf der weiter südlich gelegenen Strecke von Tongoy zu den Kupfergruben von Tamaya auf, wenig später führte die Linie bis ins nahe gelegene Provinzstädtchen Ovalle. ${ }^{20}$ Für beide Eisenbahngesellschaften sind die Betriebsberichte mit den Listen ihrer Aktionäre für mehrere Jahre überliefert. Leider findet sich in den Geschäftsberichten der Bahngesellschaften kein Hinweis auf die Nennwerte der von beiden Gesellschaften emittierten Aktien. Der Besitz an Eigenkapital der einzelnen Aktionäre lässt sich also nur als relative Größe fassen, indem ihr Besitz ins Verhältnis zur Gesamtzahl emittierter Aktien gesetzt wird. Für die Eisenbahngesellschaft von Coquimbo sind die Listen der Aktionäre für die Jahre 1868 und 1869 überliefert. 195 Aktionäre lebten in Chile, 23 in Großbritannien. Anhand der Nachnamen lassen sich zumindest Rückschlüsse auf die Herkunft der Aktionäre ziehen.

\footnotetext{
19 Anuario Estádistico de la República de Chile 1862 (Santiago de Chile 1862), S. 464.

${ }^{20}$ Luis Valenzuela Enríquez, "El transporte de Minerales. Mineros y Mineria en una Región del Norte Chico": Boletín Minero 1161 (Santiago de Chile 2007), S. 20-35.
} 
Die Aktionäre mit iberischen Namen stammten entweder aus Familien, die bereits in der Kolonialzeit oder nach dem Unabhängigkeitskrieg nach Chile ausgewandert waren. Der Anteil von Spaniern, die in Chile als Agenten für nichtchilenische Merchant Banken oder auf eigene Rechnung Geschäfte machten, dürfte hingegen minimal gewesen sein. Zum einen spielten spanische Merchant Banken in der postkolonialen Ökonomie Chiles kaum eine Rolle, zum anderen machte es beispielsweise für britische Institute wenig Sinn, sich spanischer anstatt chilenischer Staatsbürger katholischer Konfession als Mittelsmänner zu bedienen. Bei den in Chile ansässigen Aktionären mit englischen, schottischen, deutschen und französischen Nachnamen handelte es sich entweder um Nichtchilenen, die ihren eigenen oder den Geschäften ihrer Mutterhäuser in dem südamerikanischen Land nachgingen, oder sie waren wie die Edwards Einwanderer, die die chilenische Staatsbürgerschaft erhalten hatten bzw. bereits deren Nachkommen. Von den 195 in Chile lebenden Aktionären hatten 70 englische oder schottische, 18 deutsche - die Londoner Merchant Bank Huth \& Gruning ist hier ausgeklammert - und zehn französische Nachnamen. Insgesamt besaßen die in Großbritannien ansässigen Aktionäre 15\% (3.579 von 24.363) aller Aktien der Bahngesellschaft von Coquimbo. 46\% (11.127) gehörten den in Chile ansässigen Aktionären mit englischen und schottischen Namen, die Brüder Edwards nicht mit inbegriffen. Auf die Aktionäre mit deutschen Nachnamen kam ein Anteil von $12 \%$ (2.939), auf die mit französischen, ohne den chilenischen Montanunternehmer Vicente Subercaseaux, 3,5\% (849). Insgesamt besaßen die in Großbritannien ansässigen Aktionäre und die in Chile lebenden mit nichtiberischen Namen, ohne die Brüder Edwards und Vicente Subercaseaux, 76,5\% des Eigenkapitals der Gesellschaft. Die drei größten Aktionäre waren Agustín Edwards Ossandón mit 2.631 (11\%), die Chilenische Versicherungsgesellschaft (Compañía Chilena de Seguridad) mit 1.575 (6,5\%) und Ossa \& Escobar mit 1.128 Aktien $(5 \%)$.

Letztere waren führende Unternehmer in der Kupfer- und Silbermontanindustrie in der Provinz Atacama. Es folgten ein gewisser Wilhelm Müller mit 1.028 (4\%), Joaquín Edwards mit 909 (4\%) und William Gibbs mit 547 (2\%) Aktien. Alle sechs besaßen zusammen 32\% (7.818 Aktien) des Eigenkapitals der Gesellschaft. ${ }^{21}$

21 "Memoria de Directorio del Ferro-Carril de Coquimbo del Segundo Semestre de 1868", presentada a la Junta General de Accionistas, el 4 de marzo de 1869, Valparaíso, 
Tabelle 4: AktionäRe der EISEnbahngesellschaft von CoQuimbo, 1868

\begin{tabular}{l|r|r}
\hline & Aktien & Anteil \\
\hline Gesamtzahl & 24.363 & $100 \%$ \\
\hline Aktionäre mit Wohnsitz in Großbritannien & 3.579 & $15 \%$ \\
Aktien im Besitz von Aktionären mit Wohnsitz in Chile & 20.784 & $95 \%$ \\
Aktionäre mit englischen und schottischen Namen mit Wohnsitz & 11.127 & $46 \%$ \\
in Chile & & \\
Aktionäre mit deutschen Namen & 2.939 & $12 \%$ \\
Aktionäre mit französischen Namen & 849 & $3,5 \%$ \\
Agustín Edwards & 2.631 & $11 \%$ \\
Chilenische Versicherungsgesellschaft & 1.575 & $6,5 \%$ \\
Ossa \& Escobar & 1.128 & $5 \%$ \\
\hline
\end{tabular}

Quelle: "Memoria de Directorio del Ferro-Carril de Coquimbo del Segundo Semestre de 1868", presentada a la Junta General de Accionistas, el 4 de marzo de 1869, Valparaíso, Imprenta del Universo de G. Helfmann, Calle de la Aduana, Nr. 104.

Wie aus der Tabelle hervorgeht, gehörten zu den führenden Besitzern der Eisenbahn von Coquimbo 1868-1869 sowohl Montanunternehmer mit chilenischer Staatsbürgerschaft, mit einem deutlichen Gewicht der anglochilenischen Familie Edwards, als auch nichtchilenische Geschäftsleute.

Für die Eisenbahngesellschaft von Tongoy sind die Geschäftsberichte für die Zeit von 1869 bis 1892 mit Ausnahme der Jahre 18781881 sowie $1883-1884$ überliefert. 1869 hatte die Gesellschaft 1.707 Aktien emittiert, bis 1870 hatte sich ihre Zahl auf insgesamt 2.200 erhöht. 1.320 der 2.200 Aktien waren in den Händen von Aktionären mit einem Besitz von wenigstens 20 dieser Wertpapiere. Von diesen „Großaktionären“ besaßen die mit iberischen Nachnamen durchschnittlich 322 Aktien, was einem Anteil von $15 \%$ am Eigenkapital der Gesellschaft entsprach. Auf Aktionäre mit wenigstens 20 Aktien mit nichtiberischen Nachnamen entfielen durchschnittlich 988 Aktien oder $45 \%$ des Eigenkapitals der Bahngesellschaft. Davon kamen durchschnittlich 660 auf Besitzer mit englischen oder schottischen Namen, was einem Anteil von 30\% entspricht. Die Aktionäre mit deutschen Nachnamen, die im Besitz von wenigstens 20 Aktien

Imprenta del Universo de G. Helfmann, Calle de la Aduana, Nr. 104. 
waren, besaßen im Durchschnitt 191 Aktien oder 9\% des Eigenkapitals der Gesellschaft. Auf die fünf Aktionäre mit französischen Namen kamen 136 Aktien oder 6,2\% des Eigenkapitals.

Die drei größten Einzelaktionäre waren Charles Green mit 139 (6,3\%), Allan Samuel mit 119 (5,4\%) und William Hennedy mit 110 (5\%). Es folgten Ferreira und Keitel mit 100 (4,5\%) und Charles Mc Culloch und George D. Paterson mit jeweils 97 Aktien $(4,4 \%)$. Urmeneta und Errázuriz, deren Hüttenwerk in Tongoy über diese Bahn mit Erz aus Tamaya versorgt wurde, besaßen im arithmetischen Mittel 87 Aktien (4\%). Die Nichtchilenen und die chilenischen Staatsbürger, welche aus anglo- und frankophonen Ländern sowie aus Mitteleuropa eingewandert waren, hatten also unter den Besitzern von Eigenkapital an der Gesellschaft ein deutliches Übergewicht.

\section{TABelle 5: AkTionÄRE Mit Wenigstens 20 AkTIEN DER EISENBAHNGESELLSCHAFT VON TONGOY}

\begin{tabular}{l|r|r}
\hline Aktien im Besitz von... & Aktien & Anteil \\
\hline & 2.200 & $100 \%$ \\
\hline Eigentümern mit wenigstens 20 Aktien & 1.320 & $60 \%$ \\
Aktionären mit englischen und schottischen Namen & 660 & $30 \%$ \\
Aktionären mit iberischen Namen & 322 & $15 \%$ \\
Aktionären mit deutschen Namen & 191 & $9 \%$ \\
Aktionären mit französischen Namen & 136 & $6,2 \%$ \\
Charles Green & 139 & $6,3 \%$ \\
Allan Samuel & 119 & $5,4 \%$ \\
William Hennedy & 110 & $5 \%$ \\
\hline
\end{tabular}

Quelle: Die in der zweiten Spalte ausgewiesenen Werte sind die arithmetischen Mittel der in den Jahresberichten der Eisenbahngesellschaft von Tongoy für die Jahre von 1869 bis 1892, mit Ausnahme der Jahre 1878, 1879, 1880, 1881, 1883 und 1884, dokumentierten Besitzstände an Aktien der Gesellschaft.

1868 gehörten dem Direktorium der Ferro-Carril de Coquimbo folgende Mitglieder an: 
Tabelle 6: Direktorium der Ferro-Carril de Coquimbo

\begin{tabular}{l|l}
\hline Präsident & David Thomas \\
\hline Vizepräsident & Ricardo Escobar \\
\hline Direktoren & $\begin{array}{l}\text { Agustin Edwards } \\
\text { Diego Sawers } \\
\text { H. H. Hammond } \\
\text { D. W. Allardice } \\
\text { Santiago Lyon }\end{array}$ \\
\hline Inspektoren & $\begin{array}{l}\text { Oskar AD. Berckemeyer } \\
\text { Jules Meric }\end{array}$ \\
\hline Sekretär des Direktoriums & Richard G. Bunster \\
\hline Bankier & David Thomas \\
\hline Ingenieur und Verwalter & Henry A. Vivian \\
\hline
\end{tabular}

Quelle: "Memoria de Directorio del Ferro-Carril de Coquimbo del Segundo Semestre de 1868", presentada a la Junta General de Accionistas, el 4 de marzo de 1869, Valparaíso, Imprenta del Universo de G. Helfmann, Calle de la Aduana, Nr. 104.

Dem Direktorium der Ferrocarril de Tongoy gehörten 1870 folgende Mitglieder an:

Tabelle 7: Direktorium der Ferrocarril de Tongoy

\begin{tabular}{l|l}
\hline Präsident & Maximiliano Errázuriz \\
\hline Direktoren der Eigentümer & $\begin{array}{l}\text { Charles Greene } \\
\text { William L. Macqueen } \\
\text { Joseph Sothers } \\
\text { Edward Cooper }\end{array}$ \\
\hline Stellvertretende Direktoren & $\begin{array}{l}\text { M. L. Keogh } \\
\text { Karl Ebbinghaus }\end{array}$ \\
\hline Inspektoren & $\begin{array}{l}\text { Friedrich Matthai } \\
\text { William Lazonby }\end{array}$ \\
\hline Sekretär & Edwin Letts \\
\hline
\end{tabular}

Quelle: Memoria del Directorio del Ferrocarril de Tongoy, primer semestre de 1870, Valparaíso, Imprenta del Mercurio. 
Anders als bei der Gesellschaft von Coquimbo lag das Eigenkapital der Eisenbahn von Tongoy vor allem in den Händen von Nichtchilenen. Über Maximiliano Errázuriz als Direktor konnten sich Urmeneta und Errázuriz jedoch trotz ihres relativ geringen Anteils am Eigenkapital der Gesellschaft ihren Einfluss auf die Geschicke des Unternehmens sichern.

War das Eigenkapital in beiden Gesellschaften zu weit mehr als $50 \%$ in den Händen von Aktionären aus Großbritannien, dem deutschsprachigen Mitteleuropa, den USA und den frankophonen Ländern, so ist der Einfluss chilenischer Unternehmer in beiden Eisenbahngesellschaften nicht zu unterschätzen. Chilenische Unternehmer behielten nicht nur einen erheblichen Einfluss auf die Geschäfte der Gesellschaften, sondern waren aufgrund ihrer guten Reputation über längere Zeiträume hinweg in der Lage, nötige Investoren zum Erwerb von Aktien zu motivieren. Sie hatten also auch bei den beiden für die Montanindustrie der Provinz Coquimbo so wichtigen Eisenbahngesellschaften ein gehöriges Wörtchen mitzureden. 
Brought to you by | Universidad de la Rioja Authenticated Download Date | 1/22/18 1:10 PM 